\title{
Effectiveness and safety of bedaquiline- containing regimens in the treatment of MDR- and XDR-TB: a multicentre study
}

\author{
Sergey E. Borisov ${ }^{1,50}$, Keertan Dheda ${ }^{2,50}$, Martin Enwerem ${ }^{3,50}$, \\ Rodolfo Romero Leyet ${ }^{4,50}$, Lia D’Ambrosio ${ }^{5,6,50}$, Rosella Centis ${ }^{5,50}$, \\ Giovanni Sotgiu (10 ${ }^{7,50}$, Simon Tiberi ${ }^{8,9,50}$, Jan-Willem Alffenaar ${ }^{10,50}$, \\ Andrey Maryandyshev ${ }^{11,50}$, Evgeny Belilovski ${ }^{1,50}$, Shashank Ganatra' ${ }^{12,50}$ \\ Alena Skrahina ${ }^{13,50}$, Onno Akkerman ${ }^{14,15}$, Alena Aleksa ${ }^{16}$, Rohit Amale ${ }^{12}$, \\ Janina Artsukevich ${ }^{16}$, Judith Bruchfeld ${ }^{17}$, Jose A. Caminero ${ }^{18,19}$, \\ Isabel Carpena Martinez ${ }^{20}$, Luigi Codecasa ${ }^{21}$, Margareth Dalcolmo ${ }^{22}$, \\ Justin Denholm ${ }^{23}$, Paul Douglas ${ }^{24}$, Raquel Duarte ${ }^{25}$, Aliasgar Esmail ${ }^{26}$, \\ Mohammed Fadul ${ }^{26}$, Alexey Filippov ${ }^{1}$, Lina Davies Forsman ${ }^{17}$, Mina Gaga ${ }^{27}$, \\ Julia-Amaranta Garcia-Fuertes ${ }^{28}$, José-María García-García (1029, \\ Gina Gualano ${ }^{30}$, Jerker Jonsson ${ }^{31}$, Heinke Kunst ${ }^{9}$, Jillian S. Lau ${ }^{32}$, \\ Barbara Lazaro Mastrapa ${ }^{33}$, Jorge Lazaro Teran Troya ${ }^{33}$, Selene Manga ${ }^{34}$, \\ Katerina Manika ${ }^{35}$, Pablo González Montaner ${ }^{36}$, Jai Mullerpattan ${ }^{12}$, \\ Suzette Oelofse ${ }^{26}$, Martina Ortelli ${ }^{37}$, Domingo Juan Palmero ${ }^{36}$, \\ Fabrizio Palmieri ${ }^{30}$, Antonella Papalia ${ }^{38}$, Apostolos Papavasileiou ${ }^{39}$, \\ Marie-Christine Payen ${ }^{40}$, Emanuele Pontali ${ }^{41}$, Carlos Robalo Cordeiro ${ }^{42}$, \\ Laura Saderi ${ }^{7}$, Tsetan Dorji Sadutshang ${ }^{43}$, Tatsiana Sanukevich ${ }^{16}$, \\ Varvara Solodovnikova ${ }^{13}$, Antonio Spanevello 44,45 , Sonam Topgyal ${ }^{43}$, \\ Federica Toscanini ${ }^{46}$, Adrian R. Tramontana ${ }^{47}$, Zarir Farokh Udwadia ${ }^{12}$, \\ Pietro Viggiani ${ }^{38}$, Veronica White ${ }^{48}$, Alimuddin Zumla ${ }^{49}$ and Giovanni \\ Battista Migliori ${ }^{5,50}$
}


ABSTRACT Large studies on bedaquiline used to treat multidrug-resistant (MDR-) and extensively drug-resistant tuberculosis (XDR-TB) are lacking. This study aimed to evaluate the safety and effectiveness of bedaquiline-containing regimens in a large, retrospective, observational study conducted in 25 centres and 15 countries in five continents.

428 culture-confirmed MDR-TB cases were analysed (61.5\% male; 22.1\% HIV-positive, 45.6\% XDRTB). MDR-TB cases were admitted to hospital for a median (interquartile range (IQR)) 179 (92-280) days and exposed to bedaquiline for 168 (86-180) days. Treatment regimens included, among others, linezolid, moxifloxacin, clofazimine and carbapenems (82.0\%, 58.4\%, 52.6\% and $15.3 \%$ of cases, respectively).

Sputum smear and culture conversion rates in MDR-TB cases were $63.6 \%$ and $30.1 \%$, respectively at 30 days, $81.1 \%$ and $56.7 \%$, respectively at 60 days; $85.5 \%$ and $80.5 \%$, respectively at 90 days and $88.7 \%$ and $91.2 \%$, respectively at the end of treatment. The median (IQR) time to smear and culture conversion was 34 (30-60) days and 60 (33-90) days. Out of 247 culture-confirmed MDR-TB cases completing treatment, $71.3 \%$ achieved success (62.4\% cured; $8.9 \%$ completed treatment), $13.4 \%$ died, $7.3 \%$ defaulted and $7.7 \%$ failed. Bedaquiline was interrupted due to adverse events in $5.8 \%$ of cases. A single case died, having electrocardiographic abnormalities that were probably non-bedaquiline related.

Bedaquiline-containing regimens achieved high conversion and success rates under different nonexperimental conditions.

Affiliations: ${ }^{1}$ Moscow Research and Clinical Center for TB Control, Moscow Government's Health Department, Moscow, Russian Federation. ${ }^{2}$ UCT Lung Institute, Division of Pulmonology, University of Cape Town, Cape Town, South Africa. ${ }^{3}$ Amity Health Consortium, Country Club Estate, Johannesburg, South Africa. ${ }^{4}$ Clinical Unit, District Clinical Specialist Team, Springbok, South Africa. ${ }^{5}$ World Health Organization Collaborating Centre for Tuberculosis and Lung Diseases, Maugeri Care and Research Institute, Tradate, Italy. ${ }^{6}$ Public Health Consulting Group, Lugano, Switzerland. ${ }^{7}$ Clinical Epidemiology and Medical Statistics Unit, Dept of Biomedical Sciences, University of Sassari, Sassari, Italy. ${ }^{8}$ Division of Infection, Royal London Hospital, Barts Health NHS Trust, London, UK. ${ }^{9}$ Blizard Institute, Barts and The London School of Medicine and Dentistry, Queen Mary University of London, London, UK. ${ }^{10}$ University of Groningen, University Medical Center Groningen, Dept of Clinical Pharmacy and Pharmacology, Groningen, The Netherlands. ${ }^{11}$ Northern State Medical University, Arkhangelsk, Russian Federation. ${ }^{12}$ Dept of Respiratory Medicine, P.D. Hinduja National Hospital and MRC, Mumbai, India. ${ }^{13}$ Republican Research and Practical Centre for Pulmonology and Tuberculosis, Minsk, Belarus. ${ }^{14}$ University of Groningen, University Medical Center Groningen, Tuberculosis Center Beatrixoord, Haren, The Netherlands. ${ }^{15}$ University of Groningen, University Medical Center Groningen, Dept of Pulmonary Diseases and Tuberculosis, Groningen, The Netherlands. ${ }^{16}$ Dept of Phthisiology, Grodno State Medical University, GRCC "Phthisiology", Grodno, Belarus. ${ }^{17}$ Unit of Infectious Diseases, Dept of Medicine, Solna, Karolinska Institute, Dept of Infectious Diseases, Karolinska University Hospital, Stockholm, Sweden. ${ }^{18}$ Pneumology Dept, Hospital General de Gran Canaria "Dr Negrin", Las Palmas de Gran Canaria, Spain. ${ }^{19}$ MDR-TB Unit, Tuberculosis Division, International Union against Tuberculosis and Lung Disease (The Union), Paris, France. ${ }^{20}$ General University Hospital Morales Meseguer, Murcia, Spain. ${ }^{21}$ TB Reference Centre, Villa Marelli Institute/Niguarda Hospital, Milan, Italy. ${ }^{22}$ Hélio Fraga Reference Center, Fiocruz/MoH, Rio de Janeiro, Brazil. ${ }^{23}$ Victorian Tuberculosis Program, Melbourne Health, Dept of Microbiology and Immunology, University of Melbourne, Peter Doherty Institute for Infection and Immunity, Melbourne, Australia. ${ }^{24} \mathrm{Health}$ Policy and Performance Branch. Health Services and Policy Division, Dept of Immigration and Border Protection, Sydney, Australia. ${ }^{25}$ National Reference Centre for MDR-TB, Hospital Centre Vila Nova de Gaia, Dept of Pneumology, Public Health Science and Medical Education Department, Faculty of Medicine, University of Porto, Porto, Portugal. ${ }^{26}$ UCT Lung Institute, Lung Infection and Immunity Unit, Division of Pulmonology, Dept of Medicine, University of Cape Town, Groote Schuur Hospital, Cape Town, South Africa.

${ }^{27} 7$ th Respiratory Medicine Dept, Athens Chest Hospital, Athens, Greece. ${ }^{28}$ Bronchiectasis Unit, Respiratory Dept, Hospital Universitario Araba, Vitoria-Gasteiz, Spain. ${ }^{29}$ Tuberculosis Research Programme, SEPAR, Barcelona, Spain. ${ }^{30}$ Respiratory Infectious Diseases Unit, National Institute for Infectious Diseases “L. Spallanzani”, IRCCS, Rome, Italy. ${ }^{31}$ National TB Surveillance Unit, Public Health Agency, Stockholm, Sweden. ${ }^{32}$ Dept of Infectious Diseases, Box Hill Hospital, Victoria, Australia. ${ }^{33}$ Harry Surtie Hospital, Upington, South Africa. ${ }^{34}$ Dept of Infectious Diseases, University National San Antonio Abad Cusco, Cusco, Perù. ${ }^{35}$ Pulmonary Dept, 'G. Papanikolaou' Hospital, Aristotle University, Thessaloniki, Greece. ${ }^{36}$ Pulmonology Division, Municipal Hospital F. J. Munĩz, Buenos Aires, Argentina. ${ }^{37}$ Pneumology Dept, University of Insubria, Varese, Italy. ${ }^{38}$ AOVV Eugenio Morelli Hospital, Reference Hospital for MDR and HIV-TB, Sondalo, Italy. ${ }^{39}$ MDR-TB Unit, Athens Chest Hospital, Ministry of Health, Athens, Greece. ${ }^{40}$ Division of Infectious Diseases, CHU Saint-Pierre, Université Libre de Bruxelles (ULB), Brussels, Belgium. ${ }^{41}$ Dept of Infectious Diseases, Galliera Hospital, Genoa, Italy. ${ }^{42}$ Coimbra Medical School, Pneumology Dept, Coimbra University Hospital, Coimbra, Portugal. ${ }^{43}$ Delek Hospital, Dharamshala, India. ${ }^{44}$ Pneumology Dept, Maugeri Care and Research Institute, Tradate, Italy. ${ }^{45}$ Dept of Clinical and Experimental Medicine, University of Insubria, Varese, Italy. ${ }^{46}$ University Hospital San Martino, Care and Research Institute, National Institute for Cancer Research, Genoa, Italy. ${ }^{47}$ Dept of Infectious Diseases, Western Hospital, Victoria, Australia. ${ }^{48}$ Dept of Respiratory Medicine, Barts Healthcare NHS Trust, London, UK. ${ }^{49}$ Division of Infection and Immunity, University College London and NIHR Biomedical Research Centre, UCL Hospitals NHS Foundation Trust, London, UK. ${ }^{50}$ These authors contributed equally.

Correspondence: G.B. Migliori, World Health Organization Collaborating Centre for Tuberculosis and Lung Diseases, Maugeri, Care and Research Institute, Via Roncaccio 16, 21049, Tradate, Italy.

E-mail: giovannibattista.miglioridicsmaugeri.it 


\section{Introduction}

480000 cases of multidrug-resistant tuberculosis (MDR-TB) and 100000 cases of rifampicin-resistant (RR)-TB eligible for MDR-TB treatment were estimated by the World Health Organization (WHO) to have occurred in 2015, with 250000 deaths [1]. Over half of the estimated MDR-TB cases occurred in India, China, the Russian Federation and the other former Soviet Union countries, as well as in South Africa [1]. Globally, $\sim 10 \%$ of the MDR-TB strains meet the criteria defining extensively drug-resistant (XDR)-TB (resistance to any fluoroquinolone and at least one second-line injectable drug) $[1,2]$.

Treatment for MDR- and XDR-TB is long, expensive and characterised by a high rate of adverse events [3-15]. The main difficulty is the identification of at least four active drugs in order to design an effective regimen $[3,5,7,10,11]$.

The prior stepwise approach based on the hierarchical use of first- and second-line anti-TB drugs classified into five groups has been recently modified by the WHO. The new classification includes four groups of drugs (A: fluoroquinolones; B: second-line injectable agents; C: other core second-line agents; and D: add-on agents, subdivided into the subgroups D1, D2 and D3) [5].

Two newly available drugs, delamanid [12-14] and bedaquiline [15-22], together with some repurposed drugs (linezolid [7, 23-26], carbapenems [27-31] and clofazimine [32-34], among others [35, 36]) are presently pivotal in ongoing scientific discussions.

The information available today on bedaquiline is still limited to phase 2 studies and small individual cohorts treated under clinical trial conditions, the largest study not exceeding 233 patients [15-21, 37]. In particular, at this present time, no study of size informs us on the effectiveness, safety and tolerability of bedaquiline, in different continents and programmatic conditions.

Given the concerns around the adverse events of bedaquiline (particularly QT prolongation, potentially at highest risk when added to the fluoroquinolones moxifloxacin, levofloxacin and oflofloxacin, clofazimine, delamanid and methadone, among others), additional evidence on its safety, apart from that provided by the registration trials, is urgently needed [38-40].

Recently, TB reference centres belonging to the International Bedaquiline Study Group (a network merging the centres belonging to the International Carbapenems Study Group (ICSG) [29-31] and the European Respiratory Society (ERS)/Asociación Latinoamericana de Tórax (ALAT) and the Brazilian Society of Respiratory Medicine collaborative projects [41, 42], coordinated by the ERS TB collaborating centre) conducted an observational study on the therapeutic contribution of bedaquiline within a background regimen (as per WHO guidelines) when treating MDR- and XDR-TB cases.

The aim of the present study is to evaluate the safety, tolerability and effectiveness of bedaquiline within background regimens in a large multicentre cohort of MDR- and XDR-TB patients treated under different conditions (programmatic, expanded access, but not recruited for experimental studies).

\section{Material and methods}

The methodological approach adopted for this study is similar to that described in previous ICSG studies [29-31]. 25 MDR-TB reference centres located in 15 countries in Africa, Asia, Western and Eastern Europe, Oceania and Southern America (Argentina, Australia (Victoria State), Belarus, Belgium, Greece, India, Italy, the Netherlands, Peru, Portugal, Russian Federation (Arkhangelsk and Moscow city), South Africa, Spain, Sweden and United Kingdom) retrospectively collected data on RR-TB and culture-confirmed MDR-TB patients aged $\geqslant 15$ years (figure 1 and online supplementary table S1).

An MDR-TB case was defined as an individual with TB disease caused by Mycobacterium tuberculosis strains phenotypically resistant to at least isoniazid and rifampicin. RR-TB cases were those diagnosed using Xpert MTB/RIF (Cepheid, Sunnyvale, CA, USA) complemented by line probe assays. XDR-TB cases were those whose disease was due to MDR M. tuberculosis strains with additional resistance to any fluoroquinolone and one of the second-line injectable drugs (i.e. amikacin, capreomycin and kanamycin) $[1,5]$.

Patients starting their treatment between January 1, 2008 and August 30, 2016 were consecutively enrolled based on their exposure to bedaquiline during the intensive and continuation phases. We included in the study only patients exposed to bedaquiline-containing regimens, without considering the date of exposure. The condition was that bedaquiline had to be added within the design of the last treatment course.

An individualised TB regimen was administered following the results of drug susceptibility testing (DST) performed by externally quality-assured laboratories [29-31]. RR-TB cases were managed according to the national guidelines in force in their respective countries (e.g. South Africa) [43]. 


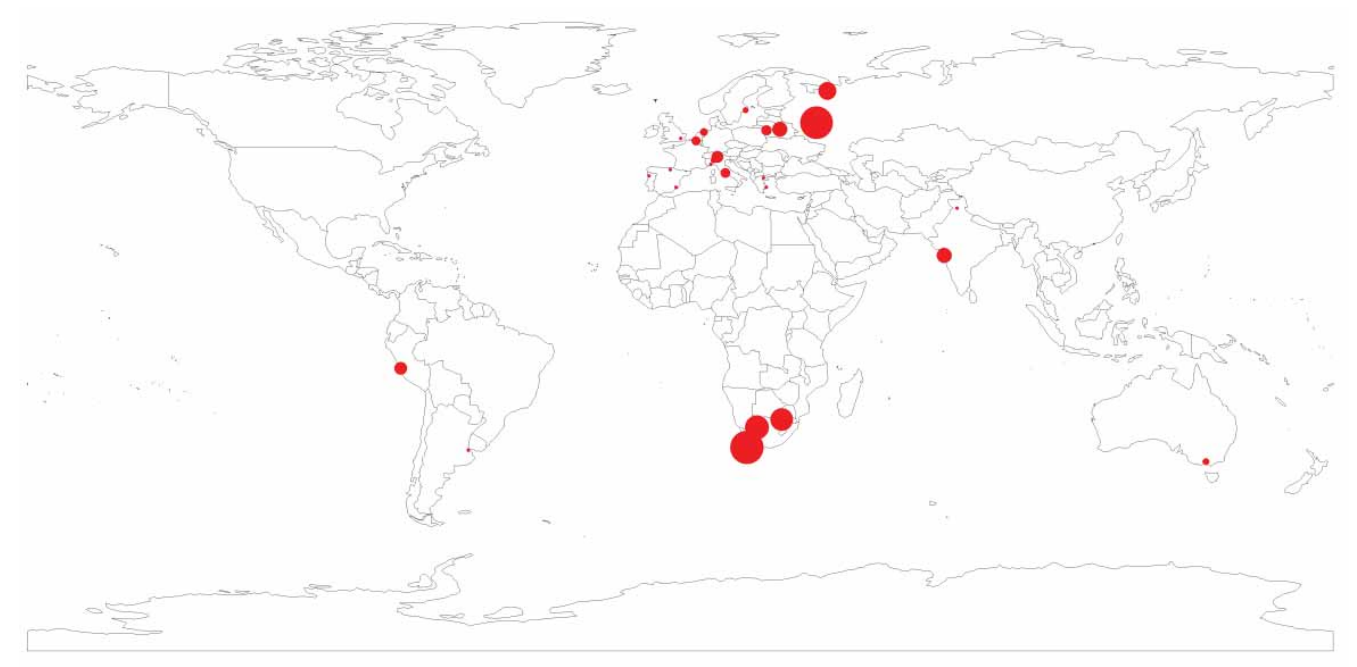

FIGURE 1 Global distribution of multidrug-resistant and extensively drug-resistant tuberculosis cases treated with regimens containing bedaquiline, 2009-2016. $n=428$ cases in 25 study sites lonline supplementary table S1).

Physicians were free to prescribe the accompanying anti-TB treatment to obtain the best possible regimen in their setting and, consequently, no specific protocol or method beyond local guidelines was followed.

Bedaquiline was administered at the recommended dose of $400 \mathrm{mg}$ once a day for 14 days, then $200 \mathrm{mg}$ three times a week for 22 weeks. It was prescribed under programmatic conditions, expanded access and compassionate use, but not under the experimental protocols of clinical trials. Adverse events were attributed to bedaquiline or to another particular drug by local attending physicians and local teams (with support of a local TB consilium, where available), without any standardised protocols or documented level of ascertainment of the causal association. A standardised classification for the severity of the adverse events was not used.

A standardised ad hoc e-form was used to collect epidemiological (i.e. age; place of birth and residence; sex; and migrant status from a TB high-incidence country), clinical (i.e. cardiac and thyroid disorders; HIV testing; HIV infection status; administration of HIV drugs; previous TB diagnosis and treatment; previous treatment outcomes; radiological findings; TB therapy and related adverse events; duration of exposure to bedaquiline, delamanid, linezolid or carbapenems; adjuvant surgery; sputum smear and culture positivity at baseline; and during treatment at 30,60 and 90 days, time to sputum smear and culture conversion; WHO treatment outcomes; and duration of hospital stay) and microbiological (i.e. DST results) data from medical records. Culture conversion rates were calculated, including in the numerator the patients with a negative culture, and in the denominator the patients completing treatment including failures, but excluding death and default outcomes.

As the latest WHO outcome definitions were introduced in 2013 and our first cohort was treated in 2008, we kept the outcome "default" instead of "lost to follow-up" [5].

With regard to the analysis of treatment outcomes, a subanalysis of MDR-TB patients who started their treatment before December 31, 2014 was performed in order to include those who could fit the definition of treatment completion and cure according to the WHO guidelines on MDR-TB treatment [5].

A homogeneous and standardised approach to DST was not possible in all the clinical centres. The percentages reported for resistance have been calculated according to the number of cases tested for (denominator) and the number of cases resistant to (numerator) a specific drug.

Ethical approval for the retrospective collection of clinical data was obtained by the coordinating centre. The centres involved in the study requested the authorisation for the treatment of their patients according to national legislation, and, accordingly, a consent form was signed by the patient and the attending physician.

The map in figure 1 was created using the rworldmap package (www.r-project.org) [44].

Qualitative and quantitative variables were summarised using percentages and medians (interquartile range (IQR)). Chi-squared or Fisher exact tests were used to compare qualitative variables, and the MannWhitney test was used to statistically compare quantitative variables. 
A p-value of $<0.05$ was considered statistically significant. Statistical computations were performed using Stata 13.0 (StataCorp, College Station, TX, USA).

\section{Results}

\section{Patient characteristics}

Demographic, epidemiological and clinical characteristics of the patients are summarised in table 1 and figure 1; safety and tolerability information on bedaquiline-containing regimens in table 2; and treatment outcome results in tables 3 and 4. 428 culture-confirmed MDR-TB patients were recruited (figure 1 and online supplementary table S1). The most prevalent sex in the cohort was male $(n=263,61.5 \%)$ and the median (IQR) age was $35(27-44)$ years. The characteristics of the 428 culture-confirmed MDR-TB patients are summarised in table 1 . There were $45(10.5 \%)$ migrants from countries with high TB incidence to those with low TB incidence.

The proportion of HIV co-infected patients was 22.1\%; their median (IQR) CD4 cell count was 269 (168470) cells $\cdot \mathrm{mm}^{-3}$ and the majority $(\mathrm{n}=92,97.9 \%)$ received antiretroviral therapy. HIV co-infection prevalence in Africa, Eastern Europe and remaining settings was 88 (46.3\%) out of 190,0 (0\%) out of 150 and $6(7.1 \%)$ out of 85 , respectively.

Pulmonary TB was diagnosed in 426 (99.5\%) out of 428 cases; the extrapulmonary locations being abdominal and the nervous system $(\mathrm{n}=2)$. The percentages of sputum smear- and culture-positive cases were $72.1 \%$ and $98.4 \%$, respectively.

Fewer than half the patients were affected by XDR-TB (195/428, 45.6\%), with a median (IQR) number of drug resistances of 3 (1-5). Overall, 334 (78.0\%) out of 428 cases had been treated previously for TB.

The prevalence of drug resistance was as follows: streptomycin 185 (94.4\%), pyrazinamide $145(70.4 \%)$, fluoroquinolones 267 (64.5\%), amikacin 131 (44.4\%), capreomycin 127 (41.6\%), kanamycin 179 (59.3\%), ethionamide 135 (59.7\%), para-aminosalicylic acid 70 (35.7\%), linezolid 4 (10.5\%), ethambutol 186 (77.5\%) and cycloserine 20 (12.3\%).

Treatment regimens included linezolid (82.0\%), clofazimine (52.6\%), moxifloxacin (58.4\%), second-line injectables (45.8\%), carbapenems (15.3\%) and ofloxacin (1.6\%).

The median (IQR) range of the administrative delay in procuring bedaquiline was 8 (0-60) days.

Patients were exposed to bedaquiline for a median (IQR) of 168 (86-180) days (table 2). Five (1.2\%) patients underwent treatment with both delamanid and bedaquiline. Adjuvant surgical therapy was performed in $55(13 \%)$ cases.

The median (IQR range) treatment duration in the cohort was 18 (10-22) months.

\section{Adverse events}

The majority of the adverse events reported were nausea, peripheral neuropathy and otovestibular toxicity. Adverse events potentially attributed to bedaquiline were reported in 80 (19.4\%) out of 413 cases where this information was provided (table 2).

In particular, $51(11.9 \%)$ out of 428 patients discontinued bedaquiline (25 (5.8\%) of whom reported adverse events); of these 26 (51\%) did so permanently.

Although we do not have the exact information on how many patients interrupted bedaquiline due to Fridericia-corrected QT interval (QTcF) increase, 24 (9.7\%) out of 247 patients experienced QTcF prolongation $>500 \mathrm{~ms}$.

One patient was started on bedaquiline with a baseline QTcF of $553 \mathrm{~ms}$, which then decreased to $536 \mathrm{~ms}$ at week 4 and $554 \mathrm{~ms}$ at week 8. A second patient, with a baseline QTcF of $352 \mathrm{~ms}$, had a transient increase ( $510 \mathrm{~ms}$ at week 3 ) followed by a decline (358 ms at week 4 ); she was palliated at home as per her request. She had had no arrhythmias at any point and no evidence to suggest that her death was related to a cardiovascular event. She achieved sputum conversion (smear and culture) prior to her death.

Figure 2 summarises the median values of the QTcF interval and its temporal trends in the cohort over the first 12 weeks of treatment.

The median (IQR) exposure to bedaquiline among the 26 patients who permanently interrupted treatment was 69 (27.5-135) days, and 85.5 (44.3-160) days in the 33 patients who died.

Out of 33 patients who died, we have QT information on 19 (57.6\%), and only one patient had a baseline QT $>500 \mathrm{~ms}$. During follow-up none of those with a normal QT at baseline showed an increase $>500 \mathrm{~ms}$. 
TABLE 1 Demographic, epidemiological and clinical characteristics of 428 culture-confirmed multidrug-resistant tuberculosis (MDR-TB) patients exposed to bedaquiline-containing regimens

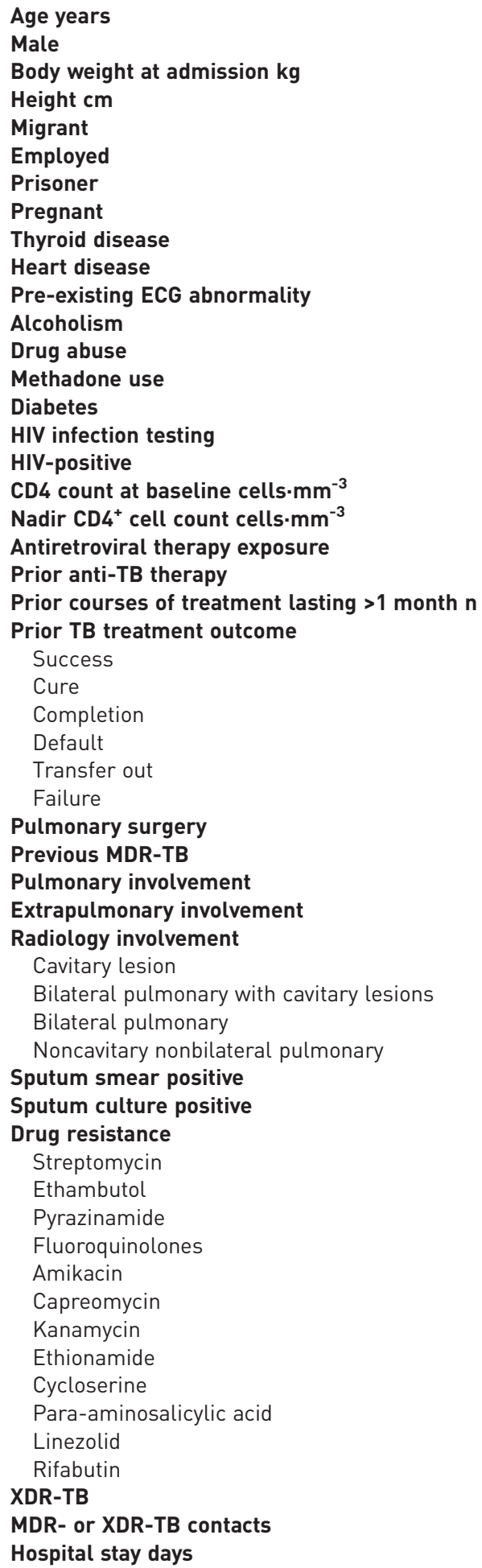

$35(27-44)$

$263 / 428(61.5)$

$56(47-65)$

$169(160-176)$

$45 / 428(10.5)$

$110 / 413(26.6)$

$23 / 413(5.6)$

$1 / 133(0.8)$

$12 / 413(2.9)$

$40 / 318(12.6)$

$13 / 318(4.1)$

$132 / 363(36.4)$

$75 / 413(18.2)$

$0 / 318(0.0)$

$26 / 413(6.3)$

425/428 (99.3)

94/425 (22.1)

269 (168-470)

222 (160-402)

92/94 (97.9)

$334 / 428$ (78.0)

2 (1-2)

68/263 (25.9)

$41 / 263(15.6)$

$27 / 263(10.3)$

$35 / 263(13.3)$

20/263 (7.6)

$140 / 263$ (53.2)

$55 / 423(13.0)$

169/317 (53.3)

426/428 (99.5)

$11 / 428(2.6)$

$81 / 331$ (24.5)

$147 / 331(44.4)$

56/331 (16.9)

47/331 (14.2)

$305 / 423(72.1)$

$421 / 428$ (98.4

$185 / 196(94.4)$

$186 / 240(77.5)$

$145 / 206(70.4)$

$267 / 414(64.5$

$131 / 295(44.4)$

$127 / 305$ (41.6)

$179 / 302$ (59.3)

$135 / 226$ (59.7)

20/163 (12.3)

70/196 (35.7)

$4 / 38(10.5)$

$32 / 35(91.4)$

$195 / 428(45.6)$

105/411 (25.6)

179 (92-280)

Data are presented as median (interquartile range) or $\mathrm{n} / \mathrm{N}$ (\%). XDR-TB: extensively drug-resistant tuberculosis. 
TABLE 2 Safety and tolerability profile of bedaquiline-containing regimens in a cohort of 428 culture-confirmed multidrug-resistant tuberculosis patients

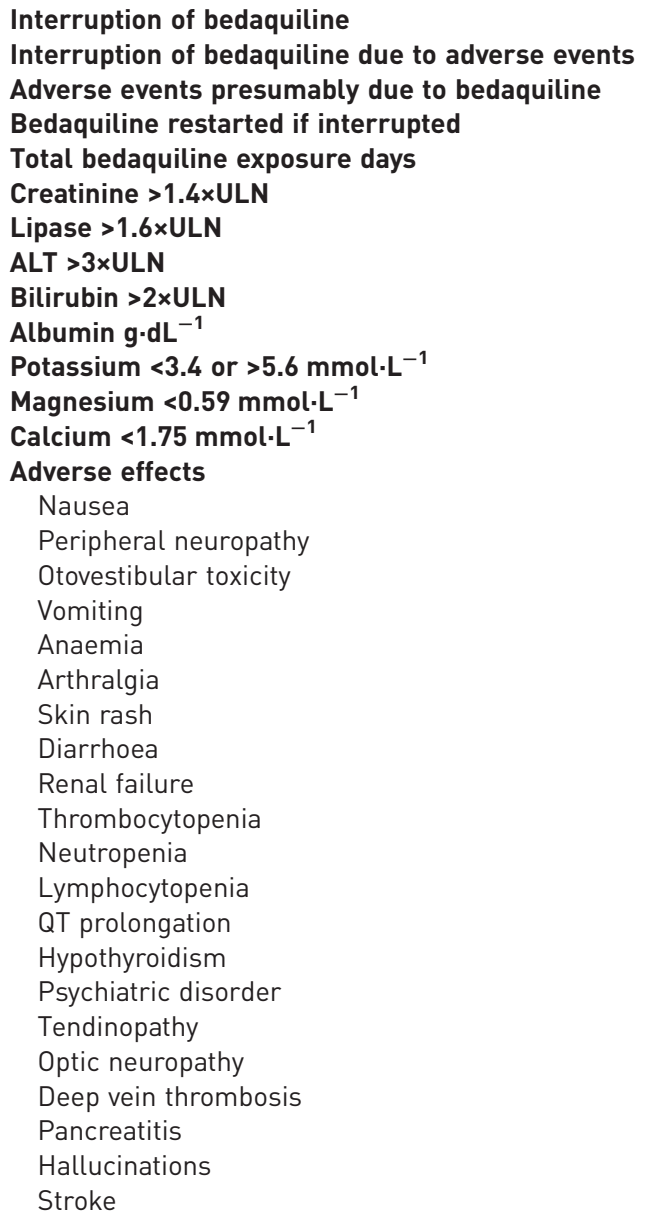

$51 / 428(11.9)$

$25 / 428(5.8)$

$80 / 213(19.4)$

$25 / 69(36.2)$

$168(86-180)$

$91 / 411(22.1)$

$1 / 239(0.4)$

92/413 (22.3)

$47 / 413(11.4)$

$36(30-40)$

$98 / 412(23.8)$

$21 / 199(10.6)$

$23 / 302(7.6)$

$130 / 413(31.5)$

96/412 (23.3)

$96 / 412(23.3)$

$87 / 411$ (21.2)

$86 / 412(20.9)$

$84 / 412(20.4)$

$63 / 412(15.3)$

$56 / 412(13.6)$

47/413 (11.4)

41/413 (9.9)

40/413 (9.7)

40/413 (9.7)

$24 / 248(9.7)$

$38 / 410(9.3)$

29/413 (7.0)

$18 / 413(4.4)$

$10 / 413(2.4)$

$7 / 412$ (1.7)

$4 / 318$ (1.3)

2/411 (0.5)

$1 / 318$ (0.3)

Data are presented as $\mathrm{n} / \mathrm{N}(\%)$ or median (interquartile range). ULN: upper limit of normal; ALT: alanine-transaminase.

According to the information available, a single case out of 33 who died had ECG abnormalities. During bedaquiline treatment the patient had a single QTcF measurement $>450 \mathrm{~ms}$, but $<500 \mathrm{~ms}$. This patient reported to the primary health centre 2 days before death complaining of weakness. The coordinating centre suggested drawing blood for electrolyte analysis, which revealed hypokalaemia. Thus, the patient was transferred to the district hospital where ECG showed premature ventricular complex bigeminy and then irreversible cardiac arrest. He died after 131 days of treatment with bedaquiline. The final ECG showed QTcF $<450 \mathrm{~ms}$. Thus, a relationship between bedaquiline use and fatal arrhythmia seems unlikely following revision of the clinical and ECG history of the patient.

Furthermore, 104 (29.9\%) out of 348 cases treated with linezolid reported adverse events attributed to this drug by the attending physician; $16(27.6 \%)$ out of 58 of them (for whom final treatment outcome was available) permanently discontinued linezolid.

\section{Sputum conversion and treatment outcomes}

Sputum smear and culture conversion rates were $63.6 \%$ and $30.1 \%$, respectively at 30 days; $81.1 \%$ and $56.7 \%$, respectively at 60 days; $85.5 \%$ and $80.5 \%$, respectively at 90 days and $88.7 \%$ and $91.2 \%$, respectively at the end of the MDR-TB treatment (for those completing it) respectively. The median (IQR) time to sputum smear and culture conversion was 34 (30-60) days and 60 (33-90) days, respectively (figure 3).

Out of 247 culture-confirmed MDR-TB cases that completed treatment, $71.3 \%$ achieved success $(62.4 \%$ cured and $8.9 \%$ completed treatment), $13.4 \%$ died, $7.3 \%$ defaulted and $7.7 \%$ failed (table 3 ). The treatment outcomes of the 130 patients initiating their treatment before December 31, 2014 are reported in table 3. 
TABLE 3 Treatment outcome and conversion rates of culture-confirmed multidrug-resistant tuberculosis (MDR-TB) patients exposed to bedaquiline-containing regimens and treatment outcomes of MDR-TB patients starting their treatment with bedaquiline-containing regimens before December 31, 2014

Bedaquiline-containing regimen

Patients $\mathbf{n}$

Sputum smear conversion 30 days

Sputum culture conversion $\mathbf{3 0}$ days

Sputum smear conversion 60 days

Sputum culture conversion 60 days

Sputum smear conversion 90 days

Sputum culture conversion 90 days

Sputum smear conversion at the end of treatment

Sputum culture conversion at the end of treatment

Time to sputum smear conversion days

Time to sputum culture conversion days

Treatment outcome

Success

Cure

Completion

Death

Default

Failure

Transfer out

Duration of treatment after MDR-TB diagnosis months

Body weight at end of treatment $\mathrm{kg}$
Bedaquiline-containing regimen started before December 31, 2014

Data are presented as $\mathrm{n} / \mathrm{N}(\%)$, median (interquartile range) or mean $\pm \mathrm{SD}$, unless otherwise stated.

TABLE 4 Treatment outcomes of 428 culture-confirmed multidrug resistant (MDR-) and extensively drug-resistant tuberculosis (XDR-TB) patients exposed to bedaquiline-containing regimens in different settings and stratified by cohort

\begin{tabular}{|c|c|c|c|c|c|c|}
\hline & Africa & Eastern Europe & Other settings & 2008-2009 & 2010-2011 & 2012-2014 \\
\hline Total cohort & 113 & 85 & 49 & & 9 & 121 \\
\hline Success & $73(64.6)$ & 65 (76.5) & $38(77.6)$ & & $5(55.6)$ & 95 (78.5) \\
\hline Cure & $73(64.6)$ & 54 (63.5) & $27(55.1)$ & & $5(55.6)$ & 81 (66.9) \\
\hline Completion & & $11(12.9)$ & $11(22.5)$ & & $0(0.0)$ & $14(11.6)$ \\
\hline Death & 27 (23.9) & $3(3.5)$ & $3(6.1)$ & & 2 (22.2) & $6(5.0)$ \\
\hline Default & $9(8.0)$ & $8(9.4)$ & $1(2.0)$ & & 2 (22.2) & 4 (3.3) \\
\hline Failure & $3(2.7)$ & $9(10.6)$ & 7 (14.3) & & $0(0.0)$ & $16(13.2)$ \\
\hline Transfer out & $1(0.9)$ & & & & & \\
\hline MDR-TB & 62 & 39 & 27 & & & \\
\hline Success & $36(58.1)$ & 28 (71.8) & 22 (81.5) & & & \\
\hline Cure & $36(58.1)$ & 20 (51.3) & $11(40.7)$ & & & \\
\hline Completion & & 8 (20.5) & $11(40.7)$ & & & \\
\hline Death & 17 (27.4) & $3(7.7)$ & 1 (3.7) & & & \\
\hline Default & 7 (11.3) & $3(7.7)$ & $1(3.7)$ & & & \\
\hline Failure & $1(1.6)$ & 5 (12.8) & $3(11.1)$ & & & \\
\hline Transfer out & $1(1.6)$ & & & & & \\
\hline XDR-TB & 51 & 46 & 22 & & & \\
\hline Success & $37(72.6)$ & 37 (80.4) & 16 (72.7) & & & \\
\hline Cure & $37(72.6)$ & 34 (73.9) & 16 (72.7) & & & \\
\hline Completion & & $3(6.5)$ & & & & \\
\hline Death & $10(19.6)$ & & $2(9.1)$ & & & \\
\hline Default & 2 (3.9) & 5 (10.9) & & & & \\
\hline Failure & 2 (3.9) & $4(8.7)$ & 4 (18.2) & & & \\
\hline Transfer out & & & & & & \\
\hline
\end{tabular}

Data are presented as $\mathrm{n}$ or $\mathrm{n}(\%)$.
$100 / 130(76.9)$

$86 / 130(66.2)$

$14 / 130(10.8)$

$8 / 130(6.2)$

$6 / 130(4.6)$

$1 / 247(0.4)$

18 (10-22)

$61.9 \pm 13.9$ 


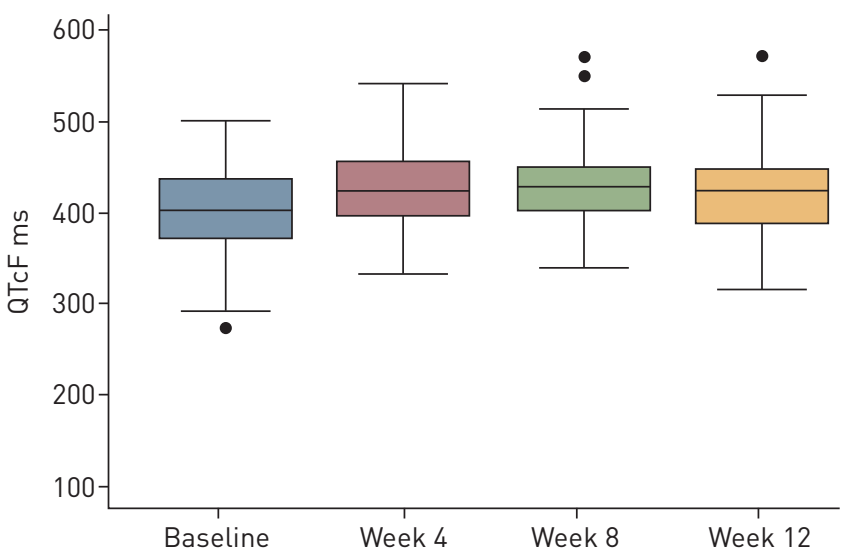

FIGURE 2 Median (interquartile range) values of the Friderica-corrected QT interval (QTcF) and its temporal trends in the cohort in the initial 12 weeks of treatment. Few patients underwent ECG after week 12.

Sputum smear and culture conversion rates at the end of treatment were not significantly different between XDR- and MDR-TB cases ( $\mathrm{p}=0.73$ and 0.96 , respectively).

The treatment success rates for MDR- and XDR-TB patients were higher in Eastern Europe and in settings other than in Africa; table 4 summarises the treatment outcome by cohort.

\section{Discussion}

The aim of the present study was to retrospectively evaluate the effectiveness, safety and tolerability of bedaquiline-containing regimens in a large observational cohort of MDR- and XDR-TB patients treated under different conditions (programmatic, expanded access, but not recruited for experimental studies).

To our knowledge, this is the largest study describing how safe, well tolerated and effective bedaquiline is within background regimens and the first in the scientific literature reporting on the nonclinical trial use of bedaquiline for the treatment of MDR- and XDR-TB patients in five continents.

In comparison with the data on individual patient data analysis (where there was a $43 \%$ success in XDR-TB) [3], in our bedaquiline-treated XDR-TB cohort the success rate was $71.3 \%$.

In a phase 2 double blind, randomised controlled trial study by Diacon et al. [16] the median time to culture conversion in 79 bedaquiline-treated MDR-TB patients was 83 days; this compares with our median time of 60 days. In the study by DiACON et al. the culture conversion at the end of 24 weeks was $79 \%$ and at 120 weeks was $58 \%$ versus $91.7 \%$ at the end of therapy in our study, and the cure rates were $58 \%$ and $62.4 \%$, respectively [16]. Regarding effectiveness, although difficult to attribute to bedaquiline, we can report that bedaquiline-containing regimens achieved culture conversion rates $>90 \%$ at the end of treatment and treatment success $>70 \%$ ( $76.7 \%$ when only calculating patients who started their treatment before December 31, 2014), higher than those observed in other MDR-TB cohorts $[3,15,17]$.

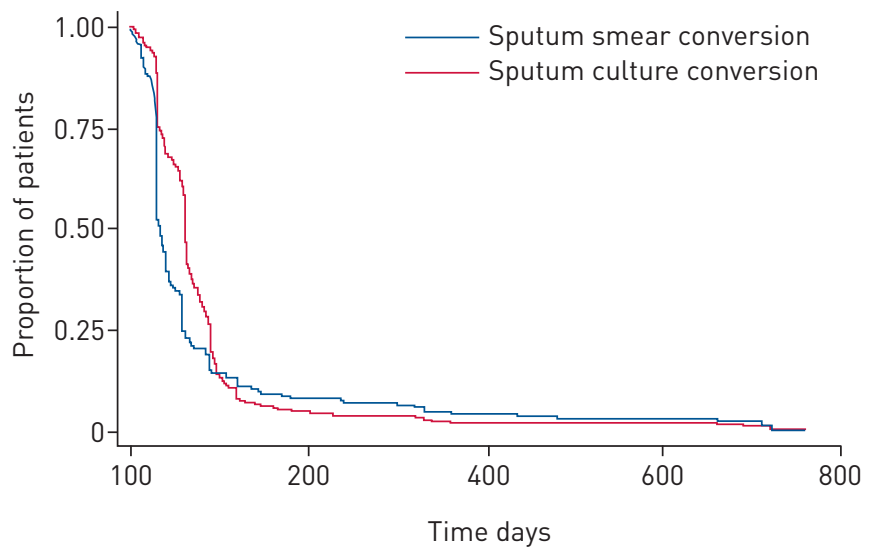

FIGURE 3 Sputum smear and culture conversion rates of 428 culture-confirmed multidrug-resistant tuberculosis patients exposed to bedaquiline-containing regimens. 
Pyм et al. [15] conducted a phase 2 trial to assess the safety and efficacy of bedaquiline in 233 patients; culture conversion was seen in $72.2 \%$ at 120 weeks, $8.6 \%$ of patients discontinued treatment and $6.9 \%$ of patients died [15].

Regarding safety, in the study by DiACON et al. [16], 13\% of patients in the bedaquiline group died (10 out of 79 versus two out of 81 in the placebo group) versus a very similar 33 (13.4\%) out of the 247 who had an evaluable outcome in our cohort.

The most frequent adverse events in our study were nausea (31.5\%), otovestibular toxicity (23.3\%), peripheral neuropathy (23.3\%), vomiting (21.2\%), anaemia (20.9\%) and arthralgia (20.4\%), their frequencies being slightly lower than those described during the licensing study by DiACON et al. (41\% nausea, $29 \%$ vomiting and 37\% arthralgia) [17]. Importantly, in the study by DiACON et al. the proportions of adverse events were similar in the treatment group versus placebo patients, suggesting that they were probably due to the background regimen. In this context, other second-line drugs such as fluoroquinolones or clofazimine might contribute to cardiologic or other adverse events [10,11,32], and invite caution and ECG monitoring.

The results of our study demonstrate that overall, bedaquiline-containing regimens achieve a relatively higher proportion of treatment success with a relatively lower proportion of adverse events within different settings than previously described, although a standardised approach for the notification of the adverse events was not implemented across the different settings (risk of underreporting).

Of note, culture conversion rates were higher than those reported in cohorts with an analogous degree of disease severity, with time to sputum smear and culture conversion identical or earlier to those observed in comparable cohorts; the proportion of treatment success was higher, and the percentage of adverse outcomes (death or failure) lower than those seen in available study cohorts with matching disease severity; adverse events due to bedaquiline leading to interruption of the drug were relatively uncommon $(5.8 \%)[10,11]$.

Our study confirms that bedaquiline-containing regimens are effective, as demonstrated by the fact that a sizeable number of patients were treated with salvage regimens due to previous treatment failure, unfavourable resistance profile, toxicity or all three.

The larger group of patients in different nonclinical trial conditions around the globe reinforces previous findings that bedaquiline is well tolerated and adverse events might be less common than previously thought.

Enthusiasm over bedaquiline and delamanid has been curtailed following concerns of potential cardiotoxicity. Both new drugs are associated with QT prolongation, which may potentially lead to arrhythmia and sudden death, a major reason why their combination has not been recommended. Moreover, the new drugs are likely to be associated with a fluoroquinolone and clofazimine, both known to prolong QT intervals. The specific role of the many drugs with QT-prolonging potential (and their summation or synergistic effect) still needs to be fully understood.

QT prolongation occurred in $9.7 \%$ of patients; interruption of bedaquiline due to adverse events occurred in $25(5.8 \%)$ patients.

Although information on QT interval was available in 64\% of cases, and the timing of their assessment not standardised, it seems the majority of cases died for non-heart-related reasons. A more accurate assessment of the adverse events (from data collection to detailed clinical investigation) will allow the evaluation of the relationship between death and QT prolongation. Unfortunately, our data are not standardised and therefore heterogeneous.

Close monitoring of drug safety should be implemented widely, particularly for rare adverse events. A comprehensive, population-level pharmacological surveillance in the post-marketing phase might allow a better assessment of the safety and tolerability profile of bedaquiline, alone or in combination with other potentially cardiotoxic anti-TB drugs [45].

The importance of implementing active TB drug-safety monitoring and management when bedaquiline is used programmatically cannot be overemphasised [45].

We underline the importance of using, in future studies, a standardised ECG monitoring protocol allowing to exclude inter- and intra-day variability in QTc measurements. For study purposes, 24-h Holter monitoring is probably the best strategy to assess the true impact of drugs on QTc. Clearly, this is not feasible under programmatic conditions.

The strengths of the study are the large cohort, the inclusion of cases from several countries (ranging from $7.7 \%$ of all patients receiving bedaquiline in the UK to $100 \%$ of bedaquiline-treated patients in Argentina, 
Australia (Victoria State), Greece, Peru, Russian Federation (Arkhangelsk oblast) and Spain) and the detailed information collected from the participating centres. The large sample size allows the comparison of treatment outcomes from different settings for the first time.

Some variables, such as the drug-resistance patterns, the number of previous anti-TB treatment cycles and HIV seroprevalence varied between the settings participating in the study.

However, the observational and retrospective design of the study has inbuilt limitations (recent guideline changes, different resource settings, different standards of care and dataset differences), so the research findings need to be confirmed by larger randomised controlled clinical trials and well-designed prospective observational studies.

Furthermore, several sources of bias may confound the reliability of the findings: missing random selectivity of the patients, heterogeneity between recruited geographic settings, different levels of completeness of collected data (especially adverse events and their severity), nonstandardised DST methods for several drugs, background regimen variability between countries which differed over time (mainly later generation fluoroquinolones), different TB/HIV co-infection prevalence and availability of antiretroviral drugs.

The effectiveness of bedaquiline might be overestimated, owing to the additive and/or synergistic role played by other effective drugs (e.g. carbapenems, linezolid, etc.) [7, 23-25, 27-31]. New experimental studies should be implemented to assess the critical effect of bedaquiline, as well as delamanid, in new combination therapies.

The different operating procedures adopted in various settings, as well as heterogeneous drug resistance patterns, may underestimate the real benefits of the bedaquiline-containing regimens.

However, stratified analyses allowed us to better assess the heterogeneity related to time and geographical factors. The new information provided by this observational study allows clinicians managing difficult-to-treat TB cases in programmatic conditions to better understand how to use bedaquiline in case the minimum number of active drugs necessary to design an effective regimen is lacking $[1,5,11]$.

Although new compounds will soon become available to support the move towards TB elimination [46], this study confirms the "core drug" characteristics of bedaquiline in the management of MDR- and XDR-TB cases even in field conditions, and eventually for use in newly designed anti-TB regimens of the future.

While it is crucial to recommend the use of bedaquiline in difficult-to-treat cases, WHO guidelines should be followed assiduously in order to limit the emergence and spread of bedaquiline-resistant $M$. tuberculosis strains.

\section{Acknowledgements}

The authors wish to thank Kerry Uebel (University of the Free State, Bloemfontein, South Africa) for supporting the study.

\section{References}

1 World Health Organization. Global Tuberculosis Report 2016. WHO/HTM/TB/2016.13 Geneva, World Health Organization, 2016.

2 Falzon D, Linh Nhat N, Jaramillo $\mathrm{E}$, et al. The global response to rifampicin-resistant tuberculosis: current situation and recent trends. Eur Respir J 2016; 48: Suppl. 60, PA1903.

3 Migliori GB, Sotgiu G, Gandhi NR, et al. Drug resistance beyond extensively drug-resistant tuberculosis: individual patient data meta-analysis. Eur Respir J 2013; 42: 169-179.

4 Diel R, Vandeputte J, de Vries G, et al. Costs of tuberculosis disease in the European Union: a systematic analysis and cost calculation. Eur Respir J 2014; 43: 554-565.

5 Falzon D, Schünemann HJ, Harausz E, et al. World Health Organization treatment guidelines for drug-resistant tuberculosis, 2016 update. Eur Respir J 2017; 49: 1602308.

6 Shean K, Streicher E, Pieterson E, et al. Drug-associated adverse events and their relationship with outcomes in patients receiving treatment for extensively drug-resistant tuberculosis in South Africa. PLoS One 2013; 8: e63057.

7 Sotgiu G, Pontali E, Migliori GB. Linezolid to treat MDR-/XDR-tuberculosis: available evidence and future scenarios. Eur Respir J 2015; 45: 25-29.

8 Tang S, Yao L, Hao X, et al. Efficacy, safety and tolerability of linezolid for the treatment of XDR-TB: a study in China. Eur Respir J 2015; 45: 161-170.

9 Lee M, Cho SN, Barry CE 3rd, et al. Linezolid for XDR-TB - final study outcomes. N Engl J Med 2015; 373: 290-291.

10 Pontali E, Sotgiu G, D’Ambrosio L, et al. Bedaquiline and multidrug-resistant tuberculosis: a systematic and critical analysis of the evidence. Eur Respir J 2016; 47: 394-402.

11 Pontali E, D'Ambrosio L, Centis R, et al. Multidrug-resistance tuberculosis and beyond: an updated analysis of the current evidence on bedaquiline. Eur Respir J 2017; 49: 1700146.

12 Esposito S, D'Ambrosio L, Tadolini M, et al. ERS/WHO Tuberculosis Consilium assistance with extensively drug-resistant tuberculosis management in a child: case study of compassionate delamanid use. Eur Respir J 2014; 44: 811-815. 
13 Skripconoka V, Danilovits M, Pehme L, et al. Delamanid improves outcomes and reduces mortality in multidrug-resistant tuberculosis. Eur Respir J 2013; 41: 1393-1400.

14 Gler MT, Skripconoka V, Sanchez-Garavito E, et al. Delamanid for multidrug-resistant pulmonary tuberculosis. N Engl J Med 2012; 366: 2151-2160.

15 Pym AS, Diacon AH, Tang SJ, et al. Bedaquiline in the treatment of multidrug- and extensively drug-resistant tuberculosis. Eur Respir J 2016; 47: 564-574.

16 Diacon AH, Pym A, Grobusch M, et al. The diarylquinoline TMC207 for multidrug-resistant tuberculosis. $N$ Engl J Med 2009; 360: 2397-2405.

17 Diacon AH, Pym A, Grobusch MP, et al. Multidrug-resistant tuberculosis and culture conversion with bedaquiline. N Engl J Med 2014; 371: 723-732.

18 Guglielmetti L, Le Dû D, Jachym M, et al. Compassionate use of bedaquiline for the treatment of multidrug-resistant and extensively drug-resistant tuberculosis: interim analysis of a French cohort. Clin Infect Dis 2015; 60: 188-194

19 Ndjeka N, Conradie F, Schnippel K, et al. Treatment of drug-resistant tuberculosis with bedaquiline in a high HIV prevalence setting: an interim cohort analysis. Int J Tuberc Lung Dis 2015; 19: 979-985.

20 Tiberi S, De Lorenzo S, Centis R, et al. Bedaquiline in MDR/XDR-TB cases: first experience on compassionate use. Eur Respir J 2014; 43: 289-292.

21 Guglielmetti L, Jaspard M, Le Dû D, et al. Long-term outcome and safety of prolonged bedaquiline treatment for multidrug-resistant tuberculosis. Eur Respir J 2017; 49: 1601799.

22 Tiberi S, D'Ambrosio L, De Lorenzo S, et al. Tuberculosis elimination, patients' lives and rational use of new drugs: revisited. Eur Respir J 2016; 47: 664-667.

23 Sotgiu G, Centis R, D'Ambrosio L, et al. Efficacy, safety and tolerability of linezolid containing regimens in treating MDR-TB and XDR-TB: systematic review and meta-analysis. Eur Respir J 2012; 40: 1430-1442.

24 Sotgiu G, Centis R, D'Ambrosio L, et al. Linezolid to treat extensively drug-resistant TB: retrospective data are confirmed by experimental evidence. Eur Respir J 2013; 42: 288-290.

25 Lee M, Lee J, Carroll MW, et al. Linezolid for treatment of chronic extensively drug-resistant tuberculosis. $N$ Engl J Med 2012; 367: 1508-1518.

26 Sotgiu G, Centis R, D’Ambrosio L, et al. Low minimal inhibitory concentrations of linezolid against multidrug-resistant tuberculosis strains. Eur Respir J 2015; 45: 287-289.

27 Tiberi S, D’Ambrosio L, De Lorenzo S, et al. Ertapenem in the treatment of multidrug-resistant tuberculosis: first clinical experience. Eur Respir J 2016; 47: 333-336.

28 van Rijn SP, van Altena R, Akkerman OW, et al. Pharmacokinetics of ertapenem in patients with multidrug-resistant tuberculosis. Eur Respir J 2016; 47: 1229-1234.

29 Tiberi S, Payen MC, Sotgiu G, et al. Effectiveness and safety of meropenem/clavulanate-containing regimens in the treatment of MDR- and XDR-TB. Eur Respir J 2016; 47: 1235-1243.

30 Tiberi S, Sotgiu G, D’Ambrosio L, et al. Effectiveness and safety of imipenem-clavulanate added to an optimized background regimen (OBR) versus OBR control regimens in the treatment of multidrug-resistant and extensively drug-resistant tuberculosis. Clin Infect Dis 2016; 62: 1188-1190.

31 Tiberi S, Sotgiu G, D'Ambrosio L, et al. Comparison of effectiveness and safety of imipenem/clavulanate-versus meropenem/clavulanate-containing regimens in the treatment of MDR- and XDR-TB. Eur Respir J 2016; 47: 1758-1766

32 Dalcolmo M, Gayoso R, Sotgiu G, et al. Effectiveness and safety of clofazimine within a standard multidrugresistant tuberculosis regimen in Brazil: first nation-wide report on over 2,500 cases. Eur Respir J 2017; 49: 1602445.

33 Tang S, Yao L, Hao X, et al. Clofazimine for the treatment of multidrug-resistant tuberculosis: prospective, multicenter, randomized controlled study in China. Clin Infect Dis 2015; 60: 1361-1367.

34 Van Deun A, Maug AK, Salim MA, et al. Short, highly effective, and inexpensive standardized treatment of multidrug-resistant tuberculosis. Am J Respir Crit Care Med 2010; 182: 684-692.

35 Winters N, Butler-Laporte G, Menzies D. Efficacy and safety of World Health Organization group 5 drugs for multidrug-resistant tuberculosis treatment. Eur Respir J 2015; 46: 1461-1470.

36 Alsaad N, Wilffert B, van Altena R, et al. Potential antimicrobial agents for the treatment of multidrug-resistant tuberculosis. Eur Respir J 2014; 43: 884-897.

37 Udwadia ZF, Amale RA, Mullerpattan JB. Initial experience of bedaquiline use in a series of drug-resistant tuberculosis patients from India. Int J Tuberc Lung Dis 2014; 18: 1315-1318.

38 Tadolini M, Lingtsang RD, Tiberi S, et al. First case of extensively drug-resistant tuberculosis treated with both delamanid and bedaquiline. Eur Respir J 2016; 48: 935-938.

39 Wallis RS. Cardiac safety of extensively drug-resistant tuberculosis regimens including bedaquiline, delamanid and clofazimine. Eur Respir J 2016; 48: 1526-1527.

40 Tadolini M, Lingtsang RD, Tiberi S, et al. Cardiac safety of extensively drug-resistant tuberculosis regimens including bedaquiline, delamanid and clofazimine. Eur Respir J 2016; 48: 1527-1529.

41 Rendon A, Fuentes Z, Torres-Duque CA, et al. Roadmap for tuberculosis elimination in Latin American and Caribbean countries: a strategic alliance. Eur Respir J 2016; 48: 1282-1287.

42 Dalcolmo M, Gayoso R, Sotgiu G, et al. Resistance profile to the drugs composing the 'shorter' regimen for multidrug-resistant TB in Brazil, 2000-2015. Eur Respir J 2017; 49: 1602309.

43 Ndjeka N, the Bedaquiline Clinical Access Programme, the Bedaquiline Implementers of the South African National TB Programme. Incorporation of Bedaquiline in the South African National TB Programme. 23rd Conference on Retroviruses and Opportunistic Infections (CROI 2016), February 22-25, 2016, Boston. Abstract \#754.

44 R Development Core Team. R: A Language and Environment for Statistical Computing. Vienna, R Foundation for Statistical Computing, 2006.

45 World Health Organization. Active Tuberculosis Drug-Safety Monitoring and Management (aDSM): Framework for Implementation. WHO/HTM/TB/2015.28. Geneva, World Health Organization, 2015

46 Lönnroth K, Migliori GB, Abubakar I, et al. Towards tuberculosis elimination: an action framework for low-incidence countries. Eur Respir J 2015; 45: 928-952. 